\title{
Study on influence of polymer and surfactant on in vitro performance of biodegradable aqueous-core nanocapsules of tenofovirdisoproxil fumarate by response surface methodology
}

\author{
Grandhi Srikar ${ }^{* 1,2}$, Avula Prameela Rani ${ }^{2}$ \\ ${ }^{1}$ School of Pharmacy, Jawaharlal Nehru Technological University Kakinada, Kakinada, India, ${ }^{2}$ College of Pharmaceutical \\ Sciences, Acharya Nagarjuna University, Guntur, India
}

\begin{abstract}
The major objective of this study was to investigate the effect of biodegradable polymer type and surfactant concentration on various characteristics viz. particle size, entrapment efficiency and drug release rate constant of aqueous core nanocapsules (ACNs) containing tenofovirdisoproxil fumarate. In this study, the nanocapsules were prepared by modified multiple emulsion technique with biodegradable polymers viz. poly(lactide-co-glycolide) of two different grades (PLGA RG502H and PLGA RG503H) and poly lactic acid (PLA R203H); and the surfactant employed was span 80. The experiments were designed under response surface methodology by employing the Design Expert software. Entrapment efficiency, particle size and drug release rate constant were taken as response variables. The prepared nanocapsules were subjected to characterization studies and the obtained results were statistically analyzed by Analysis of Variance (ANOVA) for response surface 2-Factorial Interaction model. ANOVA studies showed that the influence of both factors on all the response variables were significant at $\mathrm{p}<0.05$. The optimized formulation was found to have the entrapment efficiency of $71.58 \%$, particle size of 252.41 $\mathrm{nm}$ and the drug release rate constant of $0.045 \mathrm{~h}^{-1}$; thus, indicating that the ACNs were obtained with finest characteristics. SEM studies showed that the particles were spherical.
\end{abstract}

Keywords: Aqueous core nanocapsules. Type of polymer. Surfactant concentration. Response surface methodology.

\section{INTRODUCTION}

Carrier mediated drug delivery systems have received a growing significant interest in the recent years due to its ability of being utilized as carriers for the drug delivery for various causes such as targeted delivery (Li et al., 2008), improving bioavailability (Jia, 2005), crossing blood brain barrier (Dufes, 2011) and overcoming the inhibitory actions of glycoprotein-P (P-gP) for permeation of drugs into the cells (Brigger, Dubernet, Couvreur, 2002). Furthermore, due to the stability and suitability of incorporating wide variety of drugs, the biological, polymeric nanoparticles have gained a major share of attention among various nanocarriers viz. liposomes, niosomesand solid lipid nanoparticles (Baratt, 2000). Nanoparticles can be manufactured from a wide variety

\footnotetext{
*Correspondence: G. Srikar. College of Pharmaceutical Sciences, Acharya Nagarjuna University, Nagarjuna Nagar, Guntur - 522510, Andhra Pradesh, India. Phone: +919491335467. E-mail: srikar.grandhi@gmail.com iD
}

of natural and synthetic polymers which may or may not be biodegradable. The use of biodegradable polymers is advantageous because they are not deposited in the body and pose no toxicity risk, as they degrade into soluble and easily excretable form. These biodegradable polymeric nanoparticles can be effectively given through intravenous route in order to control the spatial and temporal delivery of the incorporated drugs. Due to their submicron size and wide possibility of modifying their surface, these can be employed to deliver drugs at varied target sites (Doppalapudi et al., 2016).

Poly (lactide-co-glycolide) (PLGA) and poly lactic acid (PLA) are the most used among various biodegradable polymers because their hydrolysis results into the simple monomers viz. lactic acid and glycolic acid which are endogenous to the body and hence have little or no toxicity. PLGA and PLA polymers can be obtained to have carboxylic acid functions as end groups so that these can have higher negative zeta potential 
(Nicolete, Santos, Faccioli, 2011; Maharana, Mohanty, Negi, 2010). Nanoparticles prepared with these polymers can have high negative zeta potential such that they can be readily engulfed by macrophages and deliver them into the reticuloendothelial system rich organs like spleen and liver (Honary, Zahir, 2013). Hence, nanoparticles prepared with polymers can be used as passive targeting carriers for delivering drugs into the liver in cases of the drugs used to treat diseases like hepatitis B. Tenofovirdisoproxil fumarate (TDF) is a prodrug that converts intracellularly into tenofovir monophosphate and tenofovirdiphospahte. It is an antiviral drug used in the treatment of HIV and chronic hepatitis B. It has a poor bioavailability of $25 \%$ after oral administration. TDF is a substrate for $\mathrm{P}-\mathrm{gP}$ and breast cancer resistance protein (BCRP) which efflux transporters that limit its availability inside the cells (Neumanova et al., 2014). These drawbacks make TDF a most desirable candidate to be developed into polymeric nanoparticulate systems which improve the bioavailability as well as avoid the effects of P-gP and BCRP.

Polymeric nanoparticles are prepared by various techniques such as nanoprecipitation, emulsion solvent evaporation, salting-out, polymerization etc. (Rao, Geckeler, 2011). Moreover, the encapsulation efficiency of these methods in case of water soluble drugs like TDF is always a concern, as these drugs tend to leach out easily. Aqueous core nanocapsules (ACNs) is one of the few techniques reported to improve the encapsulation efficiency of these drugs (Vrignaud et al., 2013). High encapsulation efficiency of ACNs is due to their unique liquid core which is optimized to have high solubility for the hydrophilic drugs and require less amount of polymer in comparison with polymer nanospheres. In vivo performance of the nanoparticles largely depends on major physicochemical characteristics like particle size, zeta potential, drug content and drug release rate constant (Crucho, Barros, 2017). Hence, it is important to investigate the influence of formulation and process variables on these characteristics of nanoparticles in order to improve their in vivo performance. Extensive literature studies revealed that numerous studies have been reported in this regard covering mainly the influence of polymer concentration, nature and composition of solvents, temperature, speed of rotation etc. But, only very few studies were reported regarding the influence of type of polymer, molecular weight of polymer and surfactant concentration on performance of nanoparticles. Budhian, Siegel and Winey (2005) studied the influence of ratio of lactic acid to glycolic acid in PLGA polymer. Sharma, Madan and Lin (2016) studied the effect of co-surfactant on the characteristics of nanoparticles. So, there is a large scope in studying the influence of these formulation parameters in order to add new data to the already existing data that might result in the development of nanoparticles with improved performance. Therefore, in this study, we aimed to prepare ACNs of TDF by modified multiple emulsion technique and explore the influence of type of polymer, molecular weight of polymer and the concentration of surfactant in the primary emulsion on entrapment efficiency, particle size and drug release rate constant. In this study, the polymers taken were PLGA of two different molecular weight grades viz. PLGA RG502H and PLGA RG503H, and PLA R203H; and the surfactant taken was span-80. The experiments for this purpose were designed according to response surface one factorial design that was repeated for every categorical factor and the obtained results were statistically analyzed using Stat Ease Design Expert software.

\section{MATERIAL AND METHODS}

\section{Materials}

TDF was obtained from Hetero Drugs Ltd (Hyderabad, India). Poly (D,L lactic acid) (RESOMER R203H/PLA R203H) and Poly (D,L lactide-co-glycolide) (RESOMER RG503H/ PLGA RG503H and RESOMER RG502H/PLGA RG502H) were obtained from SigmaAldrich (Mumbai, India). Poly vinyl alcohol was obtained from MecrkSpecialitiesPvt Ltd (Mumbai, India). Pluronic F-68, Span 80 and Chloroform were obtained from S.D Fine Chemicals (Mumbai, India). All other chemicals used were of reagent grade.

\section{Methods}

\section{Fourier transform infrared spectroscopy}

The physicochemical compatibility of TDF with PLA R203H, PLGA RG503H and PLGA RG502H used in this work were carried out by subjecting it to IR spectral studies using Fourier Transform Infrared (FT-IR) Spectrophotometer. The samples were prepared by mixing the $100 \mathrm{mg}$ of TDF with $100 \mathrm{mg}$ of each polymer and prepared as pellets with $\mathrm{KBr}$ using the hydraulic press. These pellets were scanned under diffuse reflectance mode and spectra were recorded in wave length region between $4000 \mathrm{~cm}^{-1}$ to $400 \mathrm{~cm}^{-1}$. Every spectrum was taken as mean of 16 scans per sample (Mohamed et al., 2017). The obtained spectrum of pure drug was compared with those of the physical mixtures of the drug and polymers. 
Preparation of TDF Aqueous Core Nanocapsules (TDF-ACNs)

- Experimental design

In this study, the concentration of surfactant in primary emulsion (w/o) and type of polymer were taken as two factors. Here, concentration of span 80 was the only numeric factor as the type of polymer is a categorical factor. So, a one factorial design was considered for the experiment. A linear model with three levels of the numeric factor $(\mathrm{A}$, surfactant in primary emulsion $-0 \%$, $0.25 \%$ and $0.5 \% \mathrm{v} / \mathrm{v}$ ) was considered and this design was repeated for every combination with categorical factor (B), which is also at three levels i.e. three types of polymers viz. PLGA RG503H, PLGA RG502H and PLA R203H. The experimental runs including combinations of factors at various levels were given in Table I. The response variables selected were entrapment efficiency, particle size and drug release rate constant.

- Preparation of TDF-ACNs

Nanocapsules of TDF were prepared by multiple emulsification method, which resulted after making modifications to the method reported by Yadav and Sawant (2010). A total of $100 \mathrm{mg}$ of TDF was dissolved in $5 \mathrm{~mL}$ of aqueous phase consisting of distilled water and co-solvent (Methanol) in the ratio of $4: 1$ with $1 \%$ polyvinyl alcohol as stabilizer. Approximately $100 \mathrm{mg}$ of polymer was dissolved in $10 \mathrm{~mL}$ of chloroform containing span 80 at a concentration of $0.0 \% / 0.25 \% / 0.5 \% \mathrm{v} / \mathrm{v}$, respectively. The aqueous phase was then added in drops to the organic phase while $r$ stirring at $12,000 \mathrm{rpm}$ for $30 \mathrm{~min}$ to form primary emulsion of w/o type. Thereafter, the above primary emulsion was added drop wise to $20 \mathrm{~mL}$ of $50 \% \mathrm{v} / \mathrm{v}$ aqueous glycerol as external phase containing $0.4 \% \mathrm{v} / \mathrm{v}$ of Pluronic F-68 under continuous stirring until the chloroform was evaporated. The obtained suspension of nanocapsules was stored in the refrigerator. Nanocapsules were then recovered from the nanodispersion by centrifugation (Thermo Scientific) for $30 \mathrm{~min}$ at $8,000 \mathrm{rpm}$ and $4{ }^{\circ} \mathrm{C}$, washed two times with distilled water to remove unentrapped drug and the pellet was collected. The supernatant was then used to estimate the unentrapped drug so as to determine the entrapment efficiency. The dispersion was finally lyophilized for $24 \mathrm{~h}$ to yield freeze-dried nanocapsules which were stored in air tight containers until further use.

\section{Characterization of TDF-ACNs}

\section{Differential Scanning Calorimetry (DSC)}

The physical state of TDF and polymers PLGA RG503H and PLA R203H inside the prepared nanocapsules were characterized by plotting DSC thermographs

TABLE I - Combinations of the selected factors according to the design and the obtained results of TDF-ACNs

\begin{tabular}{|c|c|c|c|c|c|c|c|c|c|}
\hline S. No. & $\begin{array}{c}\text { Formulation } \\
\text { code }\end{array}$ & $\begin{array}{l}\text { Run } \\
\text { order }\end{array}$ & $\begin{array}{c}\text { Concentration } \\
\text { of span } 80 \\
(\% \mathrm{v} / \mathrm{v})\end{array}$ & Type of polymer & $\begin{array}{c}\text { Entrapment } \\
\text { efficiency } \\
(\%)\end{array}$ & $\begin{array}{l}\text { Loading } \\
\text { efficiency } \\
(\%)\end{array}$ & $\begin{array}{c}\text { Particle } \\
\text { size } \\
(\mathrm{nm})\end{array}$ & $\begin{array}{c}\text { Zeta } \\
\text { potential } \\
(\mathbf{m V})\end{array}$ & $\begin{array}{c}\text { Release } \\
\text { rate } \\
\text { constant } \\
\left(\mathbf{h}^{-1}\right)\end{array}$ \\
\hline 1 & F1 & 1 & 0 & PLGA RG503H & 64.08 & 27.11 & 274.3 & -27.6 & 0.055 \\
\hline 2 & $\mathrm{~F} 1_{\mathrm{R}}$ & 9 & 0 & PLGA RG503H & 65.49 & 27.70 & 271.7 & -25.8 & 0.054 \\
\hline 3 & $\mathrm{~F} 2$ & 4 & 0.25 & PLGA RG503H & 69.17 & 29.26 & 265.3 & -26.9 & 0.051 \\
\hline 4 & F3 & 3 & 0.5 & PLGA RG503H & 74.25 & 31.41 & 258.6 & -28.1 & 0.047 \\
\hline 5 & $\mathrm{~F} 3_{\mathrm{R}}$ & 5 & 0.5 & PLGA RG503H & 73.86 & 31.24 & 255.1 & -29.3 & 0.046 \\
\hline 6 & F4 & 12 & 0 & PLGA RG502H & 58.21 & 24.62 & 250.6 & -23.4 & 0.085 \\
\hline 7 & $\mathrm{~F} 4_{\mathrm{R}}$ & 15 & 0 & PLGA RG502H & 57.49 & 24.32 & 249.8 & -21.9 & 0.083 \\
\hline 8 & F5 & 10 & 0.25 & PLGA RG502H & 66.15 & 27.98 & 244.3 & -22.7 & 0.069 \\
\hline 9 & F6 & 6 & 0.5 & PLGA RG502H & 68.83 & 29.12 & 242.5 & -24.2 & 0.057 \\
\hline 10 & $\mathrm{~F} 6_{\mathrm{R}}$ & 11 & 0.5 & PLGA RG502H & 70.58 & 29.86 & 238.9 & -22.8 & 0.058 \\
\hline 11 & F7 & 2 & 0 & PLA R203H & 60.13 & 25.44 & 271.2 & -18.3 & 0.052 \\
\hline 12 & $\mathrm{~F} 7_{\mathrm{R}}$ & 7 & 0 & PLA R203H & 62.27 & 26.34 & 269.7 & -20.1 & 0.054 \\
\hline 13 & F8 & 14 & 0.25 & PLA R203H & 65.96 & 27.90 & 258.1 & -18.9 & 0.049 \\
\hline 14 & F9 & 8 & 0.5 & PLA R203H & 70.35 & 29.76 & 249.3 & -21.5 & 0.046 \\
\hline 15 & $\mathrm{~F} 9_{\mathrm{R}}$ & 13 & 0.5 & PLA R203H & 71.52 & 30.25 & 250.4 & -19.5 & 0.045 \\
\hline
\end{tabular}


(Mainardes, Gremião, Evangelista, 2006). The sample was weighed, crimped into an aluminum pan and analyzed at a scanning temperature range from 20 to $250^{\circ} \mathrm{C}$ at the heating rate of $10^{\circ} \mathrm{C} / \mathrm{min}$ in nitrogen atmosphere. The thermographs of pure TDF, PLGA RG503H and PLA R203H were compared with those of TDF nanocapsules with PLGA RG503H as well as PLA R203H.

\section{Particle size and zeta potential}

The particle size and zeta potential of the prepared TDF-ACNs were determined by dynamic light scattering (DLS) technique using ZetaSizer Nano-ZS90 (Malvern Instruments, UK) (Asasutjaritet al., 2013). The measurements were carried out at an equilibrated temperature of $25^{\circ} \mathrm{C}$ and at a fixed scattering angle of $90^{\circ}$. The samples were tolerably diluted with distilled water before measurement, and measurements were made in triplicate for every sample.

\section{Scanning Electron Microscopy (SEM)}

The shape and surface characterization of nanocapsules were observed under Scanning Electron Microscope (ZEOL JSM-5610). The dispersion of nanocapsules was sufficiently diluted and a small drop was mounted directly on the SEM sample stub, using doublesided sticking tape, and coated with gold film (thickness $200 \mathrm{~nm}$ ) under reduced pressure (0.001 Torr) and it was thereafter photographed.

\section{Entrapment efficiency and drug loading}

Colloidal suspension of TDF-ACNs was centrifuged for $30 \mathrm{~min}$ at $8,000 \mathrm{rpm}$ and $4^{\circ} \mathrm{C}$. The solid pellet was washed twice with water to remove the unentrapped drug. Then, the supernatant was collected and subjected to analysis by UV-Visible Spectrophotometer at $\lambda_{\max }$ of $260 \mathrm{~nm}$ to determine the amount of drug unentrapped, from which the amount of drug entrapped was calculated (Soma et al., 2017). The entrapment efficiency and drug loading were calculated using the following formula

$$
\text { Entrapment efficiency }(\%)=\frac{\text { amount of drug entrapped }}{\text { theoretical amount of drug }} \times 100
$$$$
\text { Drug loading }(\%)=\frac{\text { amount of drug entrapped }}{\begin{array}{c}
\text { total amount of drug } \\
\text { and polymer taken }
\end{array}} \times 100
$$

In vitro drug release studies

In vitro release studies were carried out by dialysis bag method using dialysis membrane (Dialysis Membrane-110; HiMedia Lab. Pvt. Ltd., Mumbai) (Gupta et al., 2013; Bohrey, Chourasiya, Pandey, 2016). The TDF-ACNs were suspended in a small quantity of water; placed in the Gelatin bag that was sealed at both ends and suspended on receptor compartment containing $100 \mathrm{ml}$ buffer solution $(0.1 \mathrm{~N} \mathrm{HCl})$ in a beaker. The medium in the receptor was agitated continuously using a magnetic stirrer at $100 \mathrm{rpm}$ and temperature was maintained at $37 \pm 0.5^{\circ} \mathrm{C}$. A total of $2 \mathrm{~mL}$ of samples of receptor compartment were taken at various time intervals over a period of $24 \mathrm{~h}$ and at each time, fresh buffer of same volume was added. The samples were analyzed spectrophotometrically at $260 \mathrm{~nm}$ after suitable dilutions to determine the amount of drug released.

\section{Drug release kinetics and mechanism}

The data obtained from the drug release studies were subjected to zero - order and first - order kinetic models for determining the drug release kinetics and also subjected to Higuchi's and Korsemeyer - Peppas models for identifying the mechanism of drug release.

\section{Design of Experiments validation and ANOVA}

The concentration of surfactant was the only numeric factor as the type of polymer was a categorical factor. So, one factorial design linear model with one center point under response surface methodology was selected as the model which was repeated for all the three categorical factors, these resulted in a total of 15 runs. The TDF-ACNs for the 15 runs were prepared and characterized. Obtained results of the response variables were analyzed by response surface polynomial 2-Factorial Interaction (2FI) model without need of any transformation. The design was validated for its suitability into the selected 2FI model by plot of predicted versus actual results of the response variables; and also by the ANOVA of classical sum of squares - Type III 2FI model.

\section{RESULTS AND DISCUSSION}

\section{FT - IR Studies}

Compatibility studies of drug and polymer were conducted by employing I.R spectral studies. IR spectra of pure Tenofovir DF and the physical mixtures of drug and polymers were showed in Figure 1. The bands for characteristic groups of the pure Tenofovir DF were observed at $1622.93 \mathrm{~cm}^{-1}$ due to $\mathrm{N}-\mathrm{H}$ bending; $1675.44 \mathrm{~cm}^{-1}$ due to stretching of imine $(\mathrm{R} 2-\mathrm{C}=\mathrm{NR} 1)$ group; $1263.23 \mathrm{~cm}^{-1}$ because of theprimary aromatic amine stretching; $1755.23 \mathrm{~cm}^{-1}$ due to carbonyl $(\mathrm{C}=\mathrm{O})$ stretching; $1184.24 \mathrm{~cm}^{-1}$ due to $\mathrm{P}=\mathrm{O}$ stretching and at 


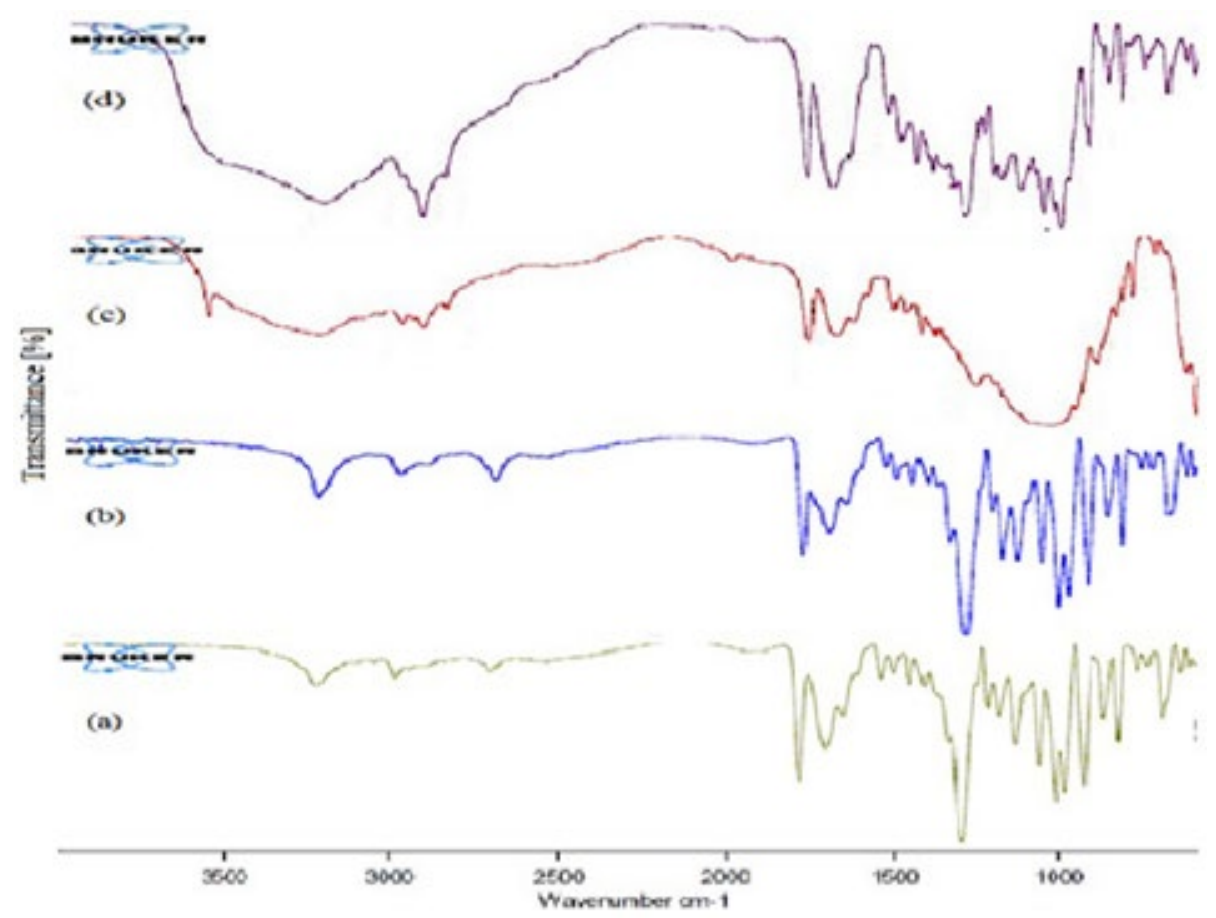

FIGURE 1 - FT - IR spectra of (a) Pure TDF; (b) Physical mixture of TDF and PLGA RG503H; (c) Physical mixture of TDF and PLGA RG502H; (d) Physical mixture of TDF and PLA R203H

$3223.48 \mathrm{~cm}^{-1}$ accounting for O-H stretching (Gomes et al., 2015; Silverstein, Webster, Kiemle, 2005). These bands accounting for the characteristic groups of TDF were also observed at the similar positions in the spectra of individual physical mixtures of TDF with the polymers viz. PLGA RG503H, PLGA RG502H and PLA R203H. These results showed that there was no interaction between TDF and the polymers as the characteristic bands were observed at the identical positions in the spectrum of pure drug and also in the spectra of physical mixtures.

\section{Preparation of TDF-ACNs}

TDF-ACNs were prepared through $\mathrm{W} / \mathrm{O} / \mathrm{W}$ emulsion by modified multiple emulsification method. The aqueous solution of TDF was emulsified in the chloroform solution of polymer to get W/O emulsion. When this emulsion was further dispersed in aqueous phase to get $\mathrm{W} / \mathrm{O} / \mathrm{W}$ emulsion and subjected to continuous stirring, chloroform got evaporated leaving the polymer to deposit on the internal aqueous phase globules. Once the chloroform got removed completely, the polymer was rigidized leaving discrete nano-sized particles with small aqueous droplets containing the drug as core and the rigid polymer as coat, so that they were called Aqueous Core Nanocapsules. The SEM studies indicated that the obtained nanocapsules were almost spherical in shape as shown in Figure 2. Here, two surfactants span 80 and
Pluronic F-68 were taken as emulsion stabilizers in the primary and secondary emulsions respectively. All the selected polymers in this study viz. PLGA RG503H, PLGA RG502H and PLA R203H were predominantly hydrophobic (Kohn, Langer, 2004; Hines, Kaplan, 2013), such that the nanocapsules obtained from these polymers have relatively hydrophobic surface which make them attacked by opsonins, that leads them to be phagocitized easily by Reticuloendothelial system (RES) rich organs, such as liver and spleen. Nanocapsules with these characteristics are better for passive targeting of drugs into liver in the treatment of liver diseases like TDF and for the treatment of hepatitis B.

\section{Experimental design}

In this work, one numeric (surfactant concentration, A) and one categorical (type of polymer, B) factor were taken, so a one factorial design repeated for every categoric factor was employed. Experiments were done according to the combinations of the factor levels suggested by the software. The obtained results were analyzed by polynomial 2FI model. The equations for the responses were obtained as

Final Equation in Terms of Coded Factors:

$\mathrm{EE}=+66.56+5.14 * \mathrm{~A}+2.81 * \mathrm{~B}[1]-2.30 * \mathrm{~B}[2]-$ $0.51 * \mathrm{AB}[1]+0.78 * \mathrm{AB}[2]$ 

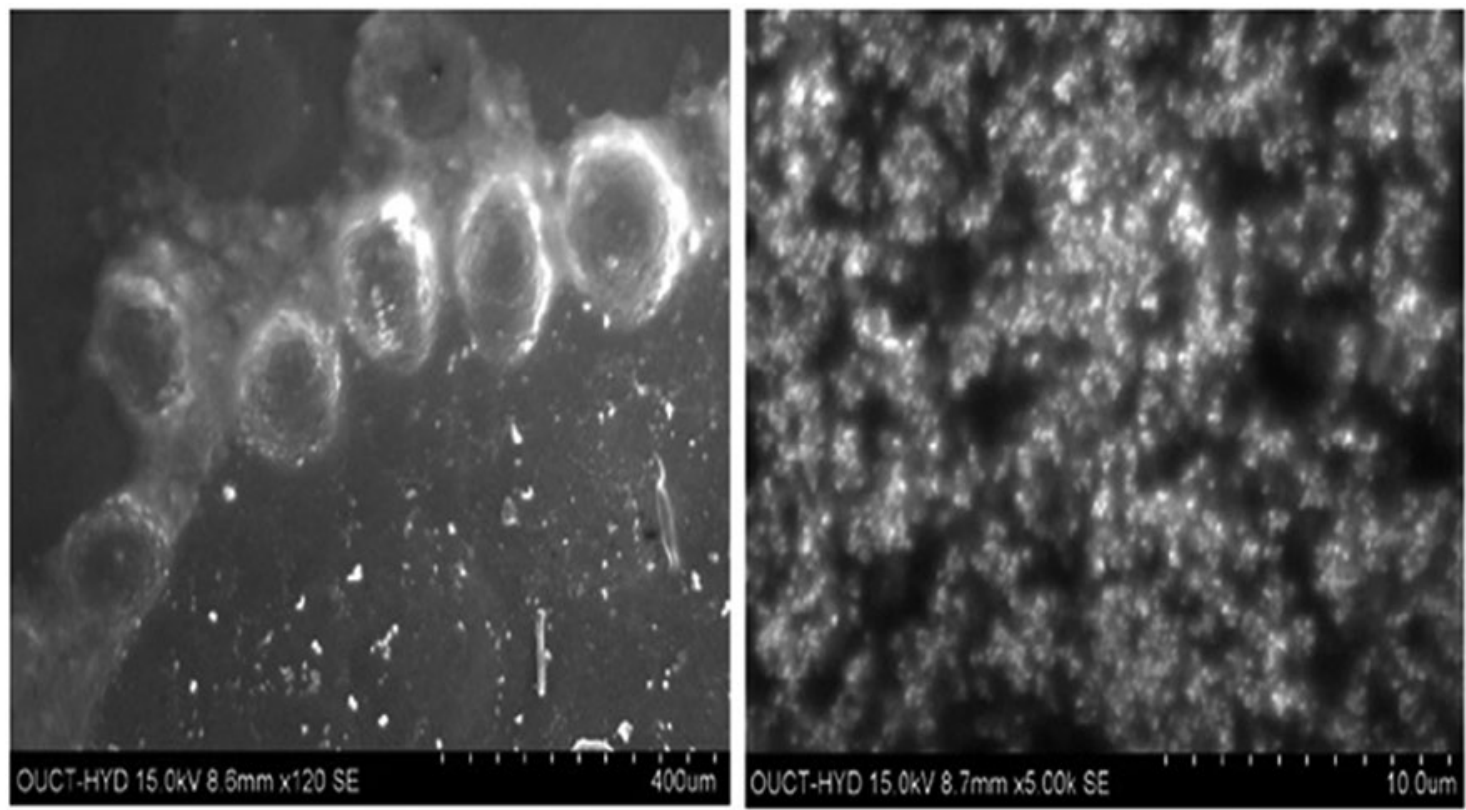

FIGURE 2 - SEM images of TDF-ACNs prepared with PLGA RG503H of formulation F3.

Particle size $=+256.65-7.71 * \mathrm{~A}+8.35 * \mathrm{~B}[1]-11.43 * \mathrm{~B}[2]$ $-0.37 * \mathrm{AB}[1]+2.96 * \mathrm{AB}[2]$

$\mathrm{k}=+0.057-0.007 * \mathrm{~A}-0.006 * \mathrm{~B}[1]+0.014 * \mathrm{~B}[2]+$ $0.003 * \mathrm{AB}[1]-0.006 * \mathrm{AB}[2]$

where, $\mathrm{EE}=$ Entrapment efficiency; $\mathrm{k}=$ drug release rate constant; $\mathrm{A}=$ surfactant concentration; $\mathrm{B}[1]$ and $\mathrm{B}[2]$ are coefficients of multi-level categorical factor.

\section{Differential Scanning Calorimetry (DSC)}

DSC analysis was performed to investigate the physical state of drug in the prepared ACNs. For this purpose, DSC spectra were taken for pure TDF; pure polymers PLA R203H and PLGA RG503H; ACNs of TDF with PLA as well as with PLGA. The spectra were shown in Figure 3. The spectra of pure drug showed a sharp endothermic peak at $118.1^{\circ} \mathrm{C}$ corresponding to its melting point temperature which is around $115^{\circ} \mathrm{C}$. But this endothermic peak was found to be absent in the spectra of TDF ACNs with either of the polymers, which indicated that the drug was in no more crystalline state in the ACNs, instead it might be in either molecular dispersion or amorphous form (Shailender et al., 2017). This might be attributed to the mode of incorporation of drug i.e. in its aqueous solution form into the nanocapsules during their preparation.

In case of the polymer PLA R203H, its pure spectra showed an endothermic peak at $55.4^{\circ} \mathrm{C}$ corresponding to its glass transition temperature $\left(T_{g}\right)$. Then, an exothermic peak at $104.8^{\circ} \mathrm{C}$ which corresponds to the re-crystallization of PLA and this was followed by another endothermic peak at $156.3{ }^{\circ} \mathrm{C}$, which indicated melting of the PLA (Tabi et al., 2010). In the spectra of TDF ACNs with PLA, the $T_{g}$ of PLA was increased and observed at $59.3^{\circ} \mathrm{C}$ which might be because of its rapid solidification during the process. The absence of further peaks of re-crystallization and its melting might be attributed to the solidification of PLA into amorphous form. The increase in $T_{g}$ was also found in case of PLGA as the endotherm in its pure spectra was observed at $48.1^{\circ} \mathrm{C}$ and in the spectra of ACNs with PLGA at $51.2{ }^{\circ} \mathrm{C}$ (Yang et al., 2016).

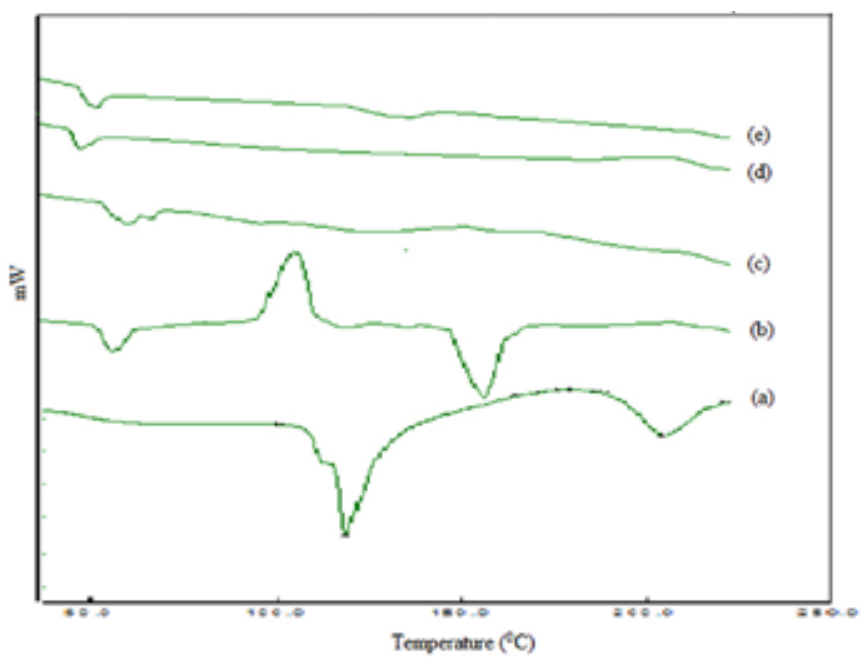

FIGURE 3 - DSC spectra of $\quad$ (a) Pure TDF; (b) Pure PLA R203H; (c) TDF-PLA R203H ACNs; (d) Pure PLGA RG503H; (e) TDF-PLGA RG503H ACNs. 


\section{Particle size}

TDF-ACNs in the size range of 242.5 to $274.3 \mathrm{~nm}$ were obtained (refer to Table I). Influence of the factors are shown in Figure 4(a). The particle size was found to decrease upon increase in the surfactant concentration. This might be attributed to the ability of surfactant to reduce the interfacial tension and hence the interfacial free energy, so that higher amount of surfactant results to an emulsion with high interfacial area, that means smaller particle size with good stability (Sinko, 2017). The results were in agreement with those reported by Rehfeld in case of benzene-in-water emulsions (Rehfeld, 1967); and also with those reported by Gupta et al.(2015) in case of solid lipid nanoparticles (Gupta et al., 2015). The particle size was found to be influenced by the type of polymer as bigger size particles were observed with PLGA RG503H followed by PLA R203H and smaller size particles were observed with PLGA RG502H. This might be attributed to the molecular weight of the polymers. The molecular weights of PLGA RG503H, PLGA RG502H and PLA $\mathrm{R} 203 \mathrm{H}$ are $24000-38000 ; 7000-17000$ and $18000-$ 28000 , respectively. The viscosity of the polymeric solution is directly proportional to its molecular weight. Under similar experimental conditions, a low viscosity polymer solution could better be dispersed in external aqueous medium with fine globule size than could a high viscous polymer solution, such that the size was reduced in the case of PLGA RG502H and increased in the order of increasing molecular weight (Ravi et al, 2008). The differences in particle size of formulations with different concentrations of surfactants and different polymers was statistically significant at $\mathrm{p}<0.05$ (as shown in Table II).

\section{Zeta potential}

The zeta potential values of prepared TDF-ACNs were found to be in the range of -18.3 to $-27.6 \mathrm{mV}$ as shown in Table I. The average zeta potential of TDFACNs was found to be $-27.54 \mathrm{mV}$ in the case of PLGA $\mathrm{RG} 503 \mathrm{H} ;-23.00 \mathrm{mV}$ in the case of PLGA RG502H; and $-19.66 \mathrm{mV}$ in the case of PLA R203H. The negative zeta potentials were attributed to the presence of free carboxylic acid as end groups on all the three selected polymers (Nicolete, dos Santos, Faccioli, 2011; Maharana, Mohanty, Negi, 2010). These results indicated that there was no influence of concentration of surfactant, but the type of polymer had influence as the zeta potential might be solely dependent on the nature of polymer that forms the mantle of the nanocapsules. The high negative zeta potential was also responsible for good physical stability in suspension form.

\section{Entrapment efficiency}

The entrapment efficiency of TDF-ACNs was found to be in the range of $58.21-73.86 \%$ (refer to Table I). Influence of the factors in this stage on entrapment efficiency areshown in Figure 4(b). The entrapment efficiency was found to be more in nanocapsules made of PLGA RG503H and decreased in the order of PLA R203H and PLGA RG502H. These results indicated that the entrapment efficiency was directly proportional to the molecular weight of the polymer. The polymer with high molecular weight would have restricted the escape of the drug encapsulated in it because of its high viscosity, resulting in high entrapment efficiency (Krishnamachari, Madan, Lin, 2007). The less particle
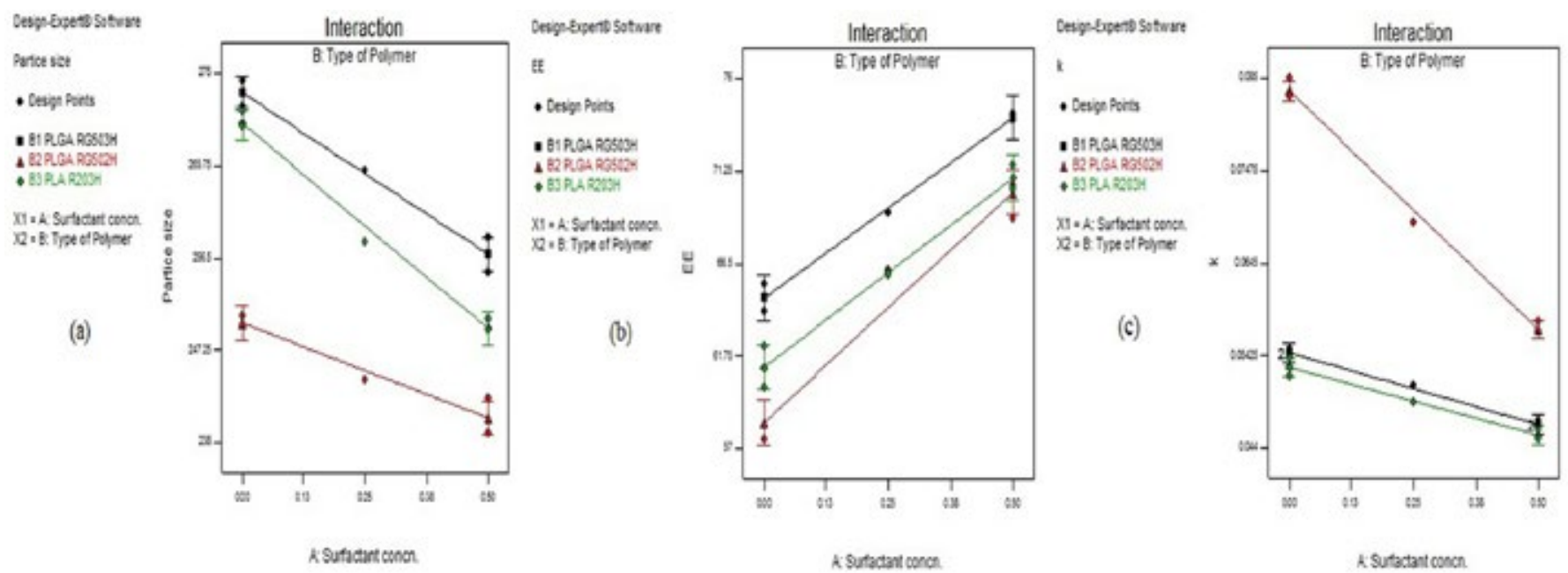

FIGURE 4 - Interaction plots showing the influence of surfactant concentration in primary emulsion and type of polymer on (a) particle size; (b) entrapment efficiency; (c) drug release rate constant. 
TABLE II - Results of ANOVA for response surface 2-factorial interaction model for the responses

\begin{tabular}{|c|c|c|c|c|c|c|c|c|}
\hline S. No. & Response & Source & $\mathbf{S S}^{\mathbf{a}}$ & $D f^{b}$ & MSS $^{\mathbf{c}}$ & F value & p-Value & Inference $^{d}$ \\
\hline \multirow{6}{*}{1} & \multirow{6}{*}{$\begin{array}{l}\text { Particle size } \\
\quad(\mathrm{nm})\end{array}$} & Model & 1825.01 & 5 & 365.00 & 145.56 & $<0.0001$ & Significant \\
\hline & & $\mathrm{A}^{\mathrm{e}}$ & 713.02 & 1 & 713.02 & 284.36 & $<0.0001$ & Significant \\
\hline & & $B^{f}$ & 1049.58 & 2 & 524.79 & 209.29 & $<0.0001$ & Significant \\
\hline & & $\mathrm{AB}$ & 62.41 & 2 & 31.21 & 12.44 & 0.0026 & Significant \\
\hline & & Residual & 22.57 & 9 & 2.51 & & & \\
\hline & & Lack of Fit & 4.53 & 3 & 1.51 & 0.50 & 0.6943 & Not significant \\
\hline \multirow{6}{*}{2} & \multirow{6}{*}{$\mathrm{EE}^{\mathrm{g}}(\%)$} & Model & 388.68 & 5 & 77.74 & 67.13 & $<0.0001$ & Significant \\
\hline & & A & 317.47 & 1 & 317.47 & 274.15 & $<0.0001$ & Significant \\
\hline & & B & 67.43 & 2 & 33.71 & 29.11 & 0.0001 & Significant \\
\hline & & $\mathrm{AB}$ & 3.78 & 2 & 1.89 & 1.63 & 0.2482 & Not significant \\
\hline & & Residual & 10.42 & 9 & 1.16 & & & \\
\hline & & Lack of Fit & 4.56 & 3 & 1.52 & 1.56 & 0.2941 & Not significant \\
\hline \multirow{6}{*}{3} & \multirow{6}{*}{$\mathrm{k}^{\mathrm{h}}\left(\mathrm{h}^{-1}\right)$} & Model & $2.23 \times 10^{-3}$ & 5 & $4.46 \times 10^{-4}$ & 461.01 & $<0.0001$ & Significant \\
\hline & & A & $5.88 \times 10^{-4}$ & 1 & $5.88 \times 10^{-4}$ & 608.28 & $<0.0001$ & Significant \\
\hline & & B & $1.41 \times 10^{-3}$ & 2 & $7.03 \times 10^{-4}$ & 727.10 & $<0.0001$ & Significant \\
\hline & & $\mathrm{AB}$ & $2.34 \times 10^{-4}$ & 2 & $1.17 \times 10^{-4}$ & 121.29 & $<0.0001$ & Significant \\
\hline & & Residual & $8.70 \times 10^{-6}$ & 9 & $9.67 \times 10^{-7}$ & & & \\
\hline & & Lack of Fit & $2.70 \times 10^{-6}$ & 3 & $9.00 \times 10^{-7}$ & 0.900 & 0.4941 & Not significant \\
\hline
\end{tabular}

Note: a Sum of Squares; b Degrees of Freedom; c Mean Sum of Squares; d p-Value less than 0.05 indicates model terms are significant; e Polymer concentration $(\% \mathrm{w} / \mathrm{w})$; e Surfactant concentration $(\% \mathrm{v} / \mathrm{v})$; f Type of polymer; g Entrapment Efficiency; h Release rate constant.

size in case of low molecular weight polymer would also be responsible for low entrapment efficiency, as the small particles could have high surface area to give more opportunity for drug escape from the nanocapsules (Yeo, Park, 2004). Hydrophobicity of the polymer can also influence the entrapment efficiency,such that the higher the hydrophobicity of polymer, the greater the resistance for escape of encapsulated drug into the outer aqueous medium. An interesting finding was observed in this work that though PLA is more hydrophobic than PLGA, high entrapment efficiency in case of PLGA RG503H demonstrated that the effect of molecular weight of the polymer might dominate the effect of hydrophobicity. The amount of Span 80, a hydrophobic surfactant, in the primary emulsion $(\mathrm{W} / \mathrm{O})$ also influenced the entrapment efficiency, such that the increase in the concentration of Span 80 led to an increase in entrapment efficiency. As the Span 80 was employed in primary emulsion (W/O), it could be deposited over the surface of the aqueous globules along with the polymer. So, upon increase in the concentration of Span 80, the nanocapsules became more hydrophobic such that the leakage of TDF into the outer aqueous medium would be minimized, resulting in increased entrapment efficiency. The influence of all the factors on entrapment efficiency was significant at $\mathrm{p}<0.05$ (as shown in Table II).

The maximum entrapment efficiency observed was $73.86 \%$. Few studies have been reported on the improvement of entrapment efficiency of hydrophilic drugs. Dordelmann et al.(2014) reported that encapsulation efficiency of nucleic acids into poly(D,Llactide-co-glycolide acid) (PLGA) nanocapsules prepared by $\mathrm{W} / \mathrm{O} / \mathrm{W}$ emulsion solvent evaporation technique was improved upto $52 \%$ by employing calcium phosphate in the primary inner phase (Dordelmann et al., 2014). Kashi et al. worked on solid/oil/water ion pairing method to encapsulate minocycline into PLGA nanoparticles and reported a maximum entrapment efficiency of $29.9 \%$ (Kashi et al., 2012). Peltonen et al reported an increase in entrapment efficiency of sodium chromoglycate into polylactide nanoparticles upto $70 \%$ by modified nanoprecipitation method (Peltonen et al., 2004). Hence, it was inferred that ACNs prepared in this study by modified multiple emulsion technique was an alternative to improving the entrapment of hydrophilic drugs into nanoparticles. 


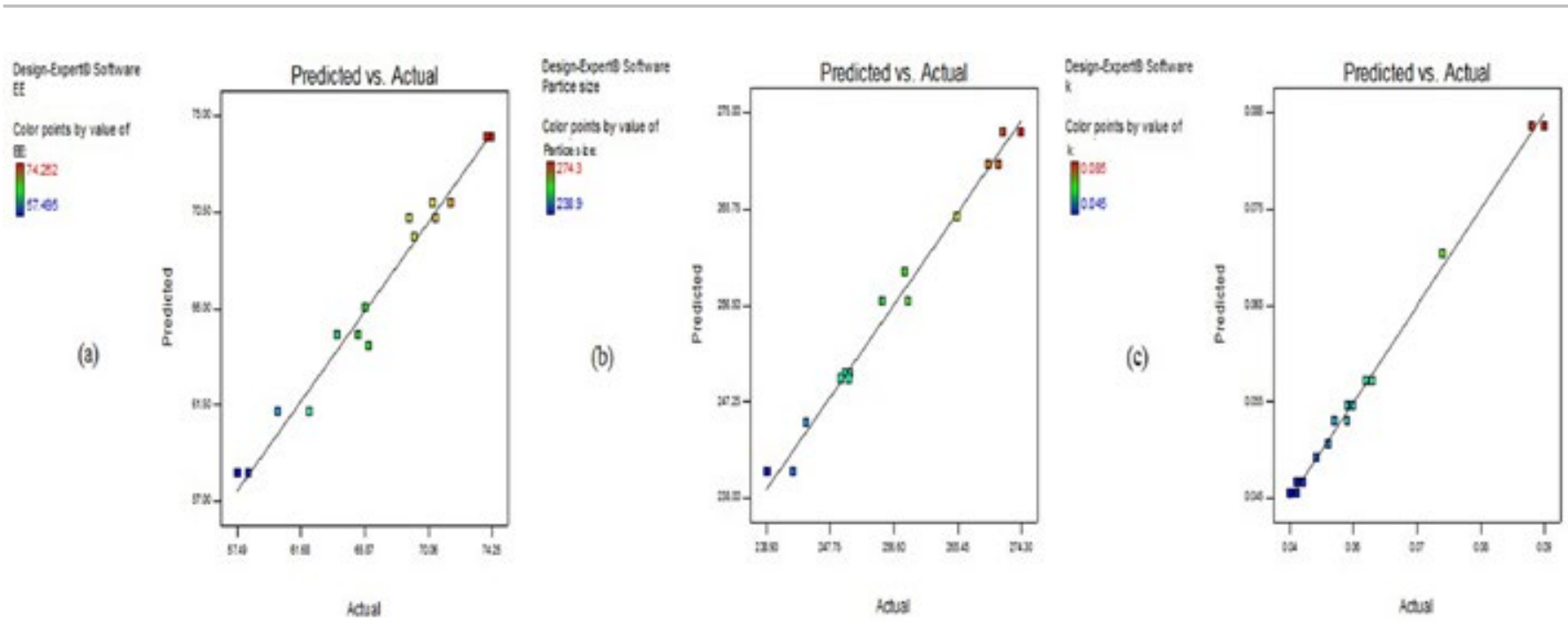

FIGURE 5 - Predicted versus actual plots for the responses (a) particle size; (b) entrapment efficiency; (c) drug release rate constant.

\section{Drug release studies}

The release rate constant of TDF from ACNs was found to be in the range of $0.045-0.058 \mathrm{~h}^{-1}$ (refer to Table I). Influence of both factors on drug release rate constant is shown in Figure 4(c). The release rate constant from nanocapsules with polymer PLGA RG502H was found to be more and this might be because of low molecular weight of this polymer among the three. The PLA or PLGA with low molecular weight could degrade or erode rapidly and release the drug encapsulated in it at high rate (Feng et al., 2015). Though, the molecular weight of PLA R203H is slightly lower than PLGA $\mathrm{RG} 503 \mathrm{H}$, the release rate constant was found to be less in TDF-ACNs with the former polymer. This might be attributed to the greater hydrophobicity of the PLA that could hinder the aqueous medium from having contact with the nanocapsules surface, thereby, resulting in decreased drug release rate constant (Ravi et al, 2008). Upon increase in the amount of Span 80 in the primary $\mathrm{W} / \mathrm{O}$ emulsion, the release rate constant was found to be decreased. As the surfactant concentration increased, the stability of the emulsion could be increased (Maa, Hsu, 1997), which might result in tight adherence of the polymer in the outer organic phase onto the surface of the internal aqueous phase globules upon evaporation of the organic solvent. So, the ACNs with high amount of Span 80 controlled the release of TDF to a greater extent. The influence of both factors on drug release rate constant was significant at $p<0.05$ (shown in Table II). Drug release from all the formulations was found to follow first - order kinetics and mechanism of drug release was found to be non - Fickian diffusion.

\section{DoE Validation and ANOVA}

The plot of predicted versus actual results of the response variables were shown in Figure 5. The close agreement between the predicted values by the design with the actual obtained values from the experiments indicated that the selected response surface 2FI model was suitable for the design employed in this study. This was also further justified by the results of ANOVA (shown in Tables II) that the model F value and insignificant lack-offit values in case of each response showed that the model was significant and suitable for the design employed in this study.

\section{CONCLUSION}

The major objective of this work was to study the influence of concentration of surfactant in primary emulsion and type of polymer on in vitro performance of ACNs of TDF that were prepared by multiple emulsion technique such that nanocapsules with superior characteristics could be developed. The findings of this work inferred that type of polymer and concentration of surfactant were found to have a significant influence on entrapment efficiency, particle size and drug release, such that these formulation variables could be successfully changed in order to obtain the ACNs with desired characteristics. In conclusion, the entrapment efficiency of hydrophilic drugs into nanoparticles could be improved by the aqueous-core nanocapsules technique.

\section{ACKNOWLEDGEMENT}

The authors are acknowledged to the authorities 
of Jawaharlal Nehru Technological University Kakinada (JNTUK), Kakinada and Acharya Nagarjuna University, Guntur for their constant support throughout the work.

\section{REFERENCES}

Asasutjarit R, Sorrachaitawatwong C, Tipchuwong N, Pouthai $\mathrm{S}$. Effect of formulation compositions on particle size and zeta potential of diclofenac sodium-loaded chitosan nanoparticles. Int J Med Health Pharmaceut Biomed Eng. 2013;7(9):568-70.

Barratt GM. Therapeutic applications of colloidal drug carriers. Pharm Sci Technol Today. 2000;3(5):163-71.

Bohrey S, Chourasiya V, Pandey A. Polymeric nanoparticles containing diazepam: preparation, optimization, characterization, in-vitro drug release and release kinetic study. Nano Converg. 2016;3(1):3.

Brigger I, Dubernet C, Couvreur P. Nanoparticles in cancer therapy and diagnosis. Adv Drug Deliv Rev. 2002;54(5):631-51.

Budhian A, Siegel SJ, Winey KI. Production of haloperidolloaded PLGA nanoparticles for extended controlled drug release of haloperidol. J Microencapsul. 2005;22(7):773-85.

Crucho CI, Barros MT. Polymeric nanoparticles: A study on the preparation variables and characterization methods. Mater Sci Eng C. 2017;80:771-84.

Doppalapudi S, Jain A, Domb AJ, Khan W. Biodegradable polymers for targeted delivery of anti-cancer drugs. Expert Opin Drug Deliv. 2016;13(6):891-909.

Dördelmann G, Kozlova D, Karczewski S, Lizio R, Knauer $\mathrm{S}$, Epple M. Calcium phosphate increases the encapsulation efficiency of hydrophilic drugs (proteins, nucleic acids) into poly (d, 1-lactide-co-glycolide acid) nanoparticles for intracellular delivery. J Mater Chem B. 2014;2(41):7250-9.

Dufès C. Brain Delivery of Peptides and Proteins. In: Chris Van Der Walle, editor. Peptide and Protein Delivery. Academic Press: Glasgow; 2011. p. 105-22.

Feng S, Nie L, Zou P, Suo J. Effects of drug and polymer molecular weight on drug release from PLGA-m PEG microspheres. J Appl Polym Sci. 2015;132(6).
Gomes EC, Mussel WN, Resende JM, Fialho SL, Barbosa J, Carignani E, Geppi M, Yoshida MI. Characterization of tenofovir disoproxil fumarate and its behavior under heating. Cryst Growth Des. 2015;15(4):1915-22.

Gupta B, Poudel BK, Pathak S, Tak JW, Lee HH, Jeong JH, Choi HG, Yong CS, Kim JO. Effects of formulation variables on the particle size and drug encapsulation of imatinib-loaded solid lipid nanoparticles. AAPS PharmSciTech. 2015;17(3):652-62.

Gupta N, Rajera R, Nagpal M, Arora S. Primaquine loaded chitosan nanoparticles for liver targeting. Pharm Nanotechnol. 2013;1(1):35-43.

Hines DJ, Kaplan DL. Poly (lactic-co-glycolic) acid-controlledrelease systems: experimental and modeling insights. Crit Rev Ther Drug Carrier Syst. 2013;30(3):257-76.

Honary S, Zahir F. Effect of zeta potential on the properties of nano-drug delivery systems-a review (Part 1). Trop J Pharm Res. 2013;12(2):255-64.

Jia L. Nanoparticle formulation increases oral bioavailability of poorly soluble drugs: approaches, experimental evidences and theory. Curr Nanosci. 2005;1(3):237-43.

Kashi TS, Eskandarion S, Esfandyari-Manesh M, Marashi SM, Samadi N, Fatemi SM, et al. Improved drug loading and antibacterial activity of minocycline-loaded PLGA nanoparticles prepared by solid/oil/water ion pairing method. Int J Nanomedicine. 2012;7:221-34.

Kohn J, Langer R. Bioresorbable and bioerodible materials. In: Ratner BD, Hoffman AS, Schoen FJ, Lemons JE, editors. Biomaterials science: an introduction to materials in medicine, 2nd ed. London: Elsevier Academic Press; 2004. p. 64-72.

Krishnamachari Y, Madan P, Lin S. Development of pH-and time-dependent oral microparticles to optimize budesonide delivery to ileum and colon. Int. J. Pharm. 2007;338(1-2):23847.

Li X, Wu Q, Chen Z, Gong X, Lin X. Preparation, characterization and controlled release of liver-targeting nanoparticles from the amphiphilic random copolymer. Polymer. 2008;49(22):4769-75.

Maa YF, Hsu CC. Effect of primary emulsions on microsphere size and protein-loading in the double emulsion process. J Microencapsul. 1997;14(2):225-41. 
Maharana T, Mohanty B, Negi YS. Preparation of poly (lactic acid) nanoparticles and optimization of the particle size. Int $\mathbf{J}$ Green Nanotechnol: Phys Chem. 2010;2(2):P100-9.

Mainardes RM, Gremião MP, Evangelista RC. Thermoanalytical study of praziquantel-loaded PLGA nanoparticles. Braz J Pharm Sci. 2006;42(4):523-30.

Mohamed AI, Abd-Motagaly AM, Ahmed OA, Amin S, Mohamed Ali AI. Investigation of drug-polymer compatibility using chemometric-assisted UV-spectrophotometry. Pharmaceutics. 2017;9(1):pii:7.

Neumanova Z, Cerveny L, Ceckova M, Staud F. Interactions of tenofovir and tenofovir disoproxil fumarate with drug efflux transporters $\mathrm{ABCB} 1, \mathrm{ABCG} 2$, and $\mathrm{ABCC} 2$; role in transport across the placenta. Aids. 2014;28(1):9-17.

Nicolete R, dos Santos DF, Faccioli LH. The uptake of PLGA micro or nanoparticles by macrophages provokes distinct in vitro inflammatory response. Int Immunopharmacol. 2011;11(10):1557-63.

Peltonen L, Aitta J, Hyvönen S, Karjalainen M, Hirvonen J. Improved entrapment efficiency of hydrophilic drug substance during nanoprecipitation of poly (I) lactide nanoparticles. AAPS PharmSciTech. 2004;5(1):115.

Rao JP, Geckeler KE. Polymer nanoparticles: preparation techniques and size-control parameters. Prog Polym Sci. 2011;36(7):887-913.

Ravi S, Peh KK, Darwis Y, Murthy BK, Singh TR, Mallikarjun C. Development and characterization of polymeric microspheres for controlled release protein loaded drug delivery system. Indian J Pharm Sci. 2008;70(3):303-9.

Rehfeld SJ. The effects of initial surfactant concentration and emulsification time upon the particle size and distribution of benzene-in-water emulsions. J Colloid Interface Sci. 1967;24(3):358-65.

Shailender J, Ravi PR, Saha P, Dalvi A, Myneni S. Tenofovir disoproxil fumarate loaded PLGA nanoparticles for enhanced oral absorption: Effect of experimental variables and in vitro, ex vivo and in vivo evaluation. Colloids Surf B Biointerfaces. 2017;158:610-9.
Sharma N, Madan P, Lin S. Effect of process and formulation variables on the preparation of parenteral paclitaxel-loaded biodegradable polymeric nanoparticles: A co-surfactant study. Asian J Pharm Sci. 2016;11(3):404-16.

Silverstein RM, Webster FX, Kiemle DJ. Spectrophotometric identification of compounds. $7^{\text {th }}$ ed. New Jersey: John Wiley and Sons; 2005.

Sinko PJ. Martin's physical pharmacy and pharmaceutical sciences. $7^{\text {th }}$ ed. New Delhi: Wolter Kluwers; 2017. p.377-8.

Soma D, Attari Z, Reddy MS, Damodaram A, Koteshwara KB. Solid lipid nanoparticles of irbesartan: preparation, characterization, optimization and pharmacokinetic studies. Braz J Pharm Sci. 2017;53(1):1-10.

Tabi T, Sajó IE, Szabó F, Luyt AS, Kovács JG. Crystalline structure of annealed polylactic acid and its relation to processing. Express Polym Lett. 2010;4(10):659-68.

Vrignaud S, Anton N, Passirani C, Benoit JP, Saulnier P. Aqueous core nanocapsules: a new solution for encapsulating doxorubicin hydrochloride. Drug Dev Ind Pharm. 2013;39(11):1706-11.

Yadav KS, Sawant KK. Modified nanoprecipitation method for preparation of cytarabine-loaded PLGA nanoparticles. AAPS PharmSciTech. 2010;11(3):1456-65.

Yang X, Trinh HM, Agrahari V, Sheng Y, Pal D, Mitra AK. Nanoparticle-based topical ophthalmic gel formulation for sustained release of hydrocortisone butyrate. AAPS PharmSciTech. 2016;17(2):294-306.

Yeo Y, Park K. Control of encapsulation efficiency and initial burst in polymeric microparticle systems. Arch Pharm Res. 2004;27(1):1-12.

Received for publication on $11^{\text {th }}$ September 2018 Accepted for publication on $06^{\text {th }}$ December 2018 